International Journal of Mechanical Engineering and Technology (IJMET)

Volume 11, Issue 11, November 2020, pp. 1-5, Article ID: IJMET_11_11_001

Available online at https://iaeme.com/Home/issue/IJMET?Volume=11\&Issue=11

ISSN Print: 0976-6340 and ISSN Online: 0976-6359

DOI: https://doi.org/10.34218/IJMET.11.11.2020.001

C IAEME Publication

Scope Database Indexed

\title{
ELECTROMAGNETIC INTERFERENCE PHILOSOPHY
}

\author{
Akbal Hanoon Yazia \\ Technical Institute of Amara, India
}

\begin{abstract}
All form of electronic equipment particularly radio frequency communication equipment suffer from electrical and magnetic inference. To electronic system such signal constitute of serious form of pollution the effect of some part of the radiofrequency $(R F)$ spectrum which is a natural. Ineffective management of the spectrum results in electromagnetic interference (EMI) in which either telecommunication systems are susceptible to service disruption from other services, or they causes and sources of EMI as well as its disruptive effects on system such as Global positioning systems, aircraft and medical devices. Various means of mitigating the effects of EMI or protecting equipment against EMI. THE wide use of electronic circuits, for communication computation and automation, makes necessary for divers circuit to operate in close proximity to each other.
\end{abstract}

Keywords: Electromagnetic, Immunity, EMI.

Cite this Article: Akbal Hanoon Yazia, Electromagnetic Interference Philosophy. International Journal of Mechanical Engineering and Technology. 11(11), 2020, pp. 1-5.

https://iaeme.com/Home/issue/IJMET?Volume $=11 \&$ Issue $=11$

\section{INTRODUCTION}

Introduction electromagnetic interference is the process by which disturbing electromagnetic energy is transmitted from an electric device to another via radiation and or conducting paths as in figure 1.1

Radiated EMI emission starts from an emitting source, propagates via a radiating path, and reached a susceptible receiver. The strength of the radiated EMI is determined by the source, and the distance between the source and the susceptor.

The basic component of EMI is an electromagnetic wave. Any source of the radiated EMI energy generates expanding spherical wave fronts, which travel in all directions from the source. At any point the wave consists of an electric field $(E)$ and a magnetic field $(H)$, which are perpendicular to each other and to the direction of propagation. The field characteristics and the relative magnitude between the $\mathrm{H}$ - field and the E-field depend upon how far away the wave is from its source and on the nature of the generating source itself. In fact, the space 
surrounding the source can be divided in two regions the region close to source is called the near, induction field, in which the field properties are determined primarily by the source characteristics. At a distance greater than $\lambda / 2 \pi$ ( $\lambda$ is the wavelength), the region is called the far, or radiation field, in this region, the properties of the field depend mainly upon the medium through which the field is propagating. The region around $\lambda / 2 \pi$ between the near and far fields is called the transition region.

EMI can be dominated by radiation or conduction, depending upon the type of coupling or propagation path involved. However, conduction always accompanies some radiation, and vice versa. Radiated interference happens when a component predominately emits energy, which is transferred to the receptor though the space. The source of the radiated interference may be part of the same system as the receptor or a completely electrically isolated unit. Conducted interference occurs when the source is connected to the receptor by power or signal cables, and the interference is transferred from one unit to the other along the cables. Conducted interference usually affects the main supply to and from the system/device and can be controlled using filters. If the conducted emissions are reduced, the relative radiated emissions are also often reduced. However, the dominant radiated interference can affect any signal path within and outside the system/device and is much more difficult to shield.

Conducted EMI emission is usually defined as undesirable electromagnetic energy coupled out of an emitter or into a susceptor via any of its respective connecting wires or cables. There are two principal modes of propagation - differential and common mode-used to characterize the conducted EMI emission. The emission of the differential mode EMI takes place between conductor pairs, which form a conventional return circuit, such as negative/positive conductors and line phase/neutral conductors. The differential EMI is the direct result of the fundamental operation of the switching converter. The emission of the common mode EMI occurs between a group of conductors and either ground or another group of conductors. The path for the common mode EMI usually includes parasitic capacitive or inductive coupling. The origin of the common mode EMI is either magnetic or electric. Common mode EMI is electrically generated when a circuit with large $\mathrm{dV} / \mathrm{dt}$ (voltage slew rate) has a significant parasitic capacitance to ground. Magnetically generated common mode EMI appears when a circuit loop with large dI/dt (current slew rate) in it has significant mutual coupling to a group of nearby conductors. In addition, there is an important energy exchange between two modes. This effect is known as differential - common mode conversion. A great the characteristic impedance of the medium. For air or free space $E / H=\mathrm{Z}_{0}=377 \Omega$, the wave is indicated to be a plane wave. In the far field, all radiated EMI waves essentially lose their curvature, and the surface containing $E$ and $H$ becomes a plane instead of a section of sphere. In the near field, the $E / H$ is determined by the characteristics of the source and distance from the source to where the field is observed. If the source has high current and low voltage $(E / H<377 \Omega)$, and may be generated by a loop, a transformer, or power lines for instance, the near field is predominantly magnetic. Conversely, if the source has low current and high voltage $(E / H>377 \Omega)$, the near field is predominantly electric. With the distance increasing away from the source, both $E$ - and $H$-fields begin to attenuate, eventually becoming the plane wave.

Depending on the characteristics of the source, the radiated EMI emission can be classified as differential mode radiation or common mode radiation. Differential mode radiation results from the flow of electromagnetic current loops within a system's structure. Common mode radiation is caused by unintentional voltage drops in a circuit, which causes some grounded parts of the circuit to rise above the referenced real ground potential. These two modes can be simply represented by the small loop radiation and dipole radiation, separately, as shown in Figure 


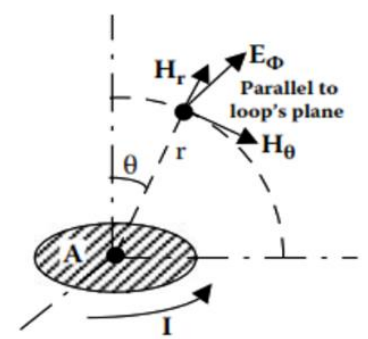

(a) Differential mode radiation from a small loop

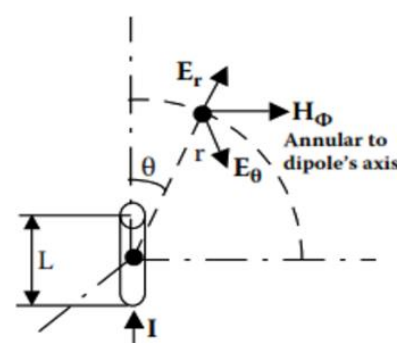

(b) Common mode radiation from a dipole

FIGURE 1.3 EMI radiation from two basic circuit configurations: (a) differential mode radiation from a small loop, (b) common-mode radiation from a dipole.

For differential mode radiation by a small loop shown in Figure $1.3 \mathrm{a}$, the $E$ and $H$ can be described approximately as (Mardiguian, 2001):

$$
\begin{gathered}
E_{\theta}(V / m)=\pi Z_{0} \frac{I A}{\lambda^{2} r} \sqrt{1+\left(\frac{\lambda}{2 \pi r}\right)^{2}} \sin \theta \\
H_{\theta}(A / m)=\pi \frac{I A}{\lambda^{2} r} \sqrt{1-\left(\frac{\lambda}{2 \pi r}\right)^{2}+\left(\frac{\lambda}{2 \pi r}\right)^{4}} \sin \theta
\end{gathered}
$$

Where $I$ is loop current (A, amper): A is loop area $\left(\mathrm{m}^{2}\right): \lambda$ is wavelength $(\mathrm{m}), \lambda=300 / f, f$ is frequency $(\mathrm{MHz}): r$ is distance to observation point $(\mathrm{m})$ : and $\mathrm{Z}_{0}$ is free space impedance, $377 \Omega$.

In the near field, $\mathrm{r}<\lambda / 2 \pi$. Equations 1.1 and 1.2 can be simplified as:

$$
\begin{aligned}
& \mathrm{E}(\mathrm{V} / \mathrm{m})=\frac{Z_{0} I A}{2 \lambda r^{2}}=0.63 \frac{I A f}{r^{2}} \\
& \mathrm{H}(\mathrm{A} / \mathrm{m})=\frac{I A}{2 \pi r^{3}}=0.08 \frac{I A}{r^{3}}
\end{aligned}
$$

Therefore the wave impedance can be obtained:

$\mathrm{Z}=E / H=7.88 \mathrm{fr}$

In the far field $r>\lambda 2 \pi$. Equations 1.1 and 1.2 can be simplified as:

$\mathrm{E}(\mathrm{V} / \mathrm{m})=\frac{Z_{0} \pi I A}{\lambda^{2} r}=0.013 \frac{I A f^{2}}{r}$

$$
\mathrm{H}(\mathrm{A} / \mathrm{m})=\frac{\pi I A}{\lambda^{2} r}=\frac{E}{120 \pi}
$$

For common mode radiation by a dipole, such as a straight wire or cable acting as an antenna, as shown in Figure 1.3 a, the $E$ and $H$ can be predicted approximately as (Mardiguian, 2001):

$$
\begin{gathered}
E_{\theta}(V / m)=z_{0} \frac{I L}{2 \lambda^{2}} \sqrt{1-\left(\frac{\lambda}{2 \pi r}\right)^{2}+\left(\frac{\lambda}{2 \pi r}\right)^{4}} \sin \theta \\
H_{\theta}(A / m)=\frac{I L}{2 \lambda r} \sqrt{1+\left(\frac{\lambda}{2 \pi r}\right)^{2}} \sin \theta
\end{gathered}
$$


Where $I$ is loop current (A. amper): $\mathrm{L}$ is dipole length $(\mathrm{m}): \lambda$ is wavelength $(\mathrm{m}), \lambda=300 / f$ is frequency $(\mathrm{MHz}) ; \mathrm{r}$ is distance to observation point $(\mathrm{m})$; and $\mathrm{Z}_{0}$ is free space impedance , $377 \Omega$.

In the near field, $r<\lambda / 2 \pi$, Equations 1.8 and 1.9 can be simplified as:

Within a complex system, EMI energy may leave a source or enter susceptible equipment by conduction, coupling, or radiation. EMI can occur between one part of the equipment and another part, such as between a power supply and nearby circuitry, or between separate equipment.

\section{ADVANCED MATERIALS AND DESIGN FOR ELECTROMAGNETIC INTERFERENCE SHIELDING}

Reducing either the $E$ - field or $H$ - field can lower EMI energy emissions and is the basis of the following suppression techniques for EMI control:

- Reduce the intensity, including the voltage and current drive levels, of the EMI source.

- Provide differential and common mode filtering for high speed signals, or use balanced differential pairs with impedance - matched signals to control the conducted EMI emission, which might come from signal lines,

- Reduce the intensity, including the voltage and current drive levels, of the EMI source.

- Provide differential and common mode filtering for high speed signals, or use balanced differential pairs with impedance - matched signals to control the conducted EMI emission, which might come from signal lines, antenna leads, power cables, and even by ground connections between EMI susceptible equipment.

- Reduce the EMI energy being coupled between components, circuits, or equipment having some mutual impedance, through which currents or voltages in one circuit can cause currents in another circuit. The mutual impedance may be conductive capacitive, or any combination of these.

- Reduce or shield radiated EMI emission from openings of any kind in equipment enclosures; ventilation, access, cable, or meter holes; around the edges of doors and hatches; drawers and panels; imperfect joints in the enclosures; and leads and cables entering a susceptible device.

- EMI shielding is always the final defense for EMC and is an effective method for controlling EMI problems.

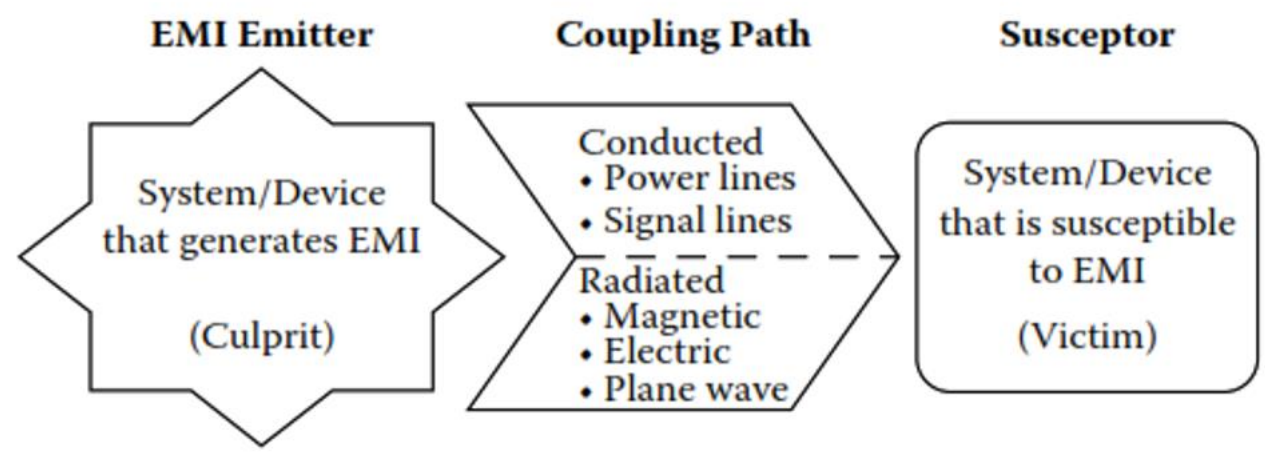

Figure 2 


\section{CONCLUSION}

To electronic system such signal constitute of serious form of pollution the effect of some part of the radiofrequency (RF) spectrum which is a natural. Ineffective management of the spectrum results in electromagnetic interference (EMI) in which either telecommunication systems are susceptible to service disruption from other services, or they causes and sources of EMI as well as its disruptive effects on system such as Global positioning systems, aircraft and medical devices. Various means of mitigating the effects of EMI or protecting equipment against EMI. THE wide use of electronic circuits, for communication computation and automation, makes necessary for divers circuit to operate in close proximity to each other.

\section{REFERENCES}

[1] Amit Bansal, Sarfaraj Ansari and Sumit Sharma 3D Electromagnetic Field Simulation of Microwave Joining of Inconel-718. International Journal of Mechanical Engineering and Technology, 8(7), 2017, pp. 1231-1237

[2] M.G. Shatrov, L.N. Golubkov, A.Yu. Dunin, P.V. Dushkin, A.L. Yakovenko, A Method of Control of Injection Rate Shape by Acting Upon Electromagnetic Control Valve of Common Rail Injector, International Journal of Mechanical Engineering and Technology 8(11), 2017, pp. 676-690.

[3] A.M. Aswin and S. Sundar, Electromagnetic Interference (Emi) and Electromagnetic Compatibility (Emc) in Real Time Applications - Survey, International Journal of Mechanical Engineering and Technology, 9(6), 2018, pp. 474-482

[4] Dr. Srinivasa Baba, V. S. S. N., Giri Raju, B. and Mrs. Srivalli Gundala. Electromagnetic Interference (EMI) Analysis for Oblique Incidence of EM Waves in Double Shields. International Journal of Electronics and Communication Engineering \& Technology, 6(11), 2015, pp. 01-09 